RESEARCH ARTICLE

\title{
Cholinergic receptor blockers affect the protective effect of Angong Niuhuang pill on neurologic deficits and intestinal injury in intracerebral hemorrhage mice
}

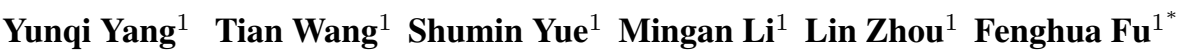 \\ ${ }^{1}$ Department of Pharmacology, School of Pharmacy, Yantai University, Yantai 264005, China
}

\section{Check for updates}

Correspondence to: Fenghua Fu, Department of Pharmacology, School of Pharmacy, Yantai University, Yantai 264005, China;

E-mail: fufenghua@sohu.com

Received: December 31, 2020;

Accepted: January 14, 2021;

Published: January 18, 2021

Citation: Yang Y, Wang T, Yue S, et al. Cholinergic receptor blockers affect the protective effect of Angong Niuhuang Pill on neurologic deficits and intestinal injury in intracerebral hemorrhage mice. $J$ Pharm Biopharm Res, 2021, 2(2): 153-160.

https://doi.org/10.25082/JPBR.2020.02.002

Copyright: (c) 2021 Fenghua Fu, et al. This is an open access article distributed under the terms of the Creative Commons Attribution License, which permits unrestricted use, distribution, and reproduction in any medium, provided the original author and source are credited.

Funding: This work was supported by National Natural Science Foundation of China (No. 81873039).

\begin{abstract}
Stroke is a devastating disease, intracerebral hemorrhage (ICH) is a devastating subtype. This study aimed to investigate whether cholinergic receptors participate in the process of Angong Niuhuang pill (ANP) improving neurological function and relieving intestinal injury in ICH mice. The mice were treated with ANP, cholinergic receptor blockers, atropine (ATR), penehyclidine hydrochloride (PHC) or methyllycaconitine (MLA). Male CD-1 mice were randomly divided into 9 groups, Sham, ICH, ANP $(0.2 \mathrm{~g} / \mathrm{kg})$, ANP plus ATR, ANP plus PHC, ANP plus MLA, ATR, PHC, MLA. ICH model is made by collagenase VII injection $(0.075 \mathrm{U})$. ANP $(0.2 \mathrm{~g} / \mathrm{kg})$ was administered by gavage after $30 \mathrm{~min}$ of ICH. MLA, ATR, PHC was given at 15 min after ICH. Neurological function was evaluated by Garcia test. Intestinal injury was observed by histological analysis. Endotoxin (ET) was measured by enzyme-linked immunosorbent assay. Compared to the Sham group, the score of Garcia test in the ICH significantly decreased. ANP increased significantly the score of ICH mice. ANP also ameliorated the intestinal injury caused by ICH. Cholinergic receptor blockers reversed partially the improvement of neurological function and intestinal injury by ANP. ANP attenuates the neurological deficits and intestinal injury in ICH mice and the protective effect of ANP may be involved in the regulation of the cholinergic system.
\end{abstract}

Keywords: Angong Niuhuang pill, intracerebral hemorrhage, neurological function, intestinal injury, cholinergic receptors

\section{Introduction}

Stroke is a devastating disease all over the world. It is often accompanied by high morbidity, mortality and disability rates [1]. According to the systematic analysis for the global burden, stroke is the leading causes of death in China in 2017 [2]. Stroke consists hemorrhagic and ischemic stroke [3]. Intracerebral hemorrhage (ICH) is the most devastating subtype of stroke, consists about $10 \%-15 \%$ of stroke [4]. ICH cause high rate of mortality and mortality.

ICH led to inflammation, cognitive dysfunction, peripheral organ injury, which lower the quality of patients' life [5-8]. Clinical studies have shown that inflammation is closely related to a poor prognosis of cerebral hemorrhage [9]. Systemic inflammation is companied with stroke and stroke also causes intestinal injury. Stress response after ICH leads to gastrointestinal ischemia and hypoxia and therefore causes the destruction of intestinal mucosa. Thereby, endotoxin (ET), intestinal bacteria, and endogenous substances enter the blood, resulting in inflammatory damage. Inflammatory mediators of circulation enter the brain to aggravate the central nervous system injury [10].

Previous work showed that stroke will lead to autonomic nerve dysfunction, which manifests as sympathetic nervous system excitation and parasympathetic nervous system inhibition [11] Some experiments show that $\beta$ receptor blocker may exert a neuroprotection in ICH patients by inhibiting sympathetic nervous system [12]. Vagus nerve is a part of parasympathetic nervous system. Stimulation of vagus nerve attenuates the secretion of tumor necrosis factor- $\alpha$ (TNF- $\alpha$ ) from macrophage and inhibits the response of systemic inflammation [13]. It reported that $\alpha 7$ nicotinic acetylcholine receptor $(\alpha 7 \mathrm{nAChR})$ plays an essential role in the process of anti-inflammation of vagus nerve stimulation [14].

There are different cholinergic receptor blockers, such as atropine (ATR) [15], penehyclidine hydrochloride (PHC) and methyllycaconitine (MLA). ATR is a non-selective antagonist of muscarinic receptors [16,17]. $\mathrm{PHC}$ is an antagonist of $\mathrm{M}_{1}$ and $\mathrm{M}_{3}$ receptor and it is manufactured in China. MLA is a selective antagonist of $\alpha 7 \mathrm{nAChR}$. 
Angong Niuhuang pill (ANP) is a famous traditional Chinese medicine which is used for the treatment of coma after stroke. In China, ANP was employed as adjuvant therapy for treating acute cerebral infarction and acute ICH [18-20]. ICH is often accompanied with intestinal mucosal injury and intestinal permeability increase. However, the role of the parasympathetic nervous system in the effects of ANP on ICH remains unclear and needs to be studied.

\section{Material and methods}

\subsection{Drugs and reagents}

Angong Niuhuang pill (Batch number: 19013256) was purchased from Beijing Tongrentang Co., LTD. PHC (Batch number: 190802) was purchased from Chengdu Lystra Pharmaceutical Co. Ltd. ATR (Batch number: 1902251) was from Tianjin Jinyao Pharmaceutical Co. LTD. MLA (Batch number: 22) was purchased from Tocris Bioscience/R\&D Systems. Mouse endotoxin enzyme-linked immunosorbent assay (ELISA) kits (Product number: ml002005) were from Shanghai Mlbio Biotechnology Co., Ltd.

\subsection{Animals and experimental design}

Male CD-1 mice (body weight 30 to $35 \mathrm{~g}$ ) were purchased from Jinan Pengyue company (Jinan, China). Animals were kept in 21 to $25^{\circ} \mathrm{C}$ on 12 light-dark cycle. Animals can get food and water freely.

The experiments were performed according to the guidelines for the use of experimental animals of Yantai University, and were approved by the Ethics Committee of Yantai University.

The mice were randomly divided into 9 groups ( $\mathrm{n}=8$ in each group): Sham, ICH, ANP $(0.2$ $\mathrm{g} / \mathrm{kg}$ ), ANP plus ATR, ANP plus PHC, ANP plus MLA, ATR, PHC, MLA. The daily dose of ANP in human is $3 \mathrm{~g} /$ day. Previous study from our laboratory demonstrated that ANP protected the neurologic function and reduced the intestinal injury in ICH mice [21]. In our pilot study, ANP at dose of $0.2 \mathrm{~g} / \mathrm{kg}$ showed the best effect of neuroprotection. ATR was administered subcutaneously at a dose of $1 \mathrm{mg} / \mathrm{kg}$. MLA was given intraperitoneally at a dose of $6 \mathrm{mg} / \mathrm{kg}$. PHC was administered intramuscularly at a dose of $1 \mathrm{mg} / \mathrm{kg}$. ATR, MLA and PHC was given at 15 min after ICH. The mice were treated with ANP by gavage at $0.5 \mathrm{~h}$ after ICH.

\subsection{ICH model}

Mice were anesthetized by intraperitoneal injection with ketamine $(80 \mathrm{mg} / \mathrm{kg})$ and xylazine $(5 \mathrm{mg} / \mathrm{kg})$. ICH model is made by infusing $0.075 \mathrm{U}$ of collagenase in right striatum according to previous report [22]. The midline of the scalp was cut. The skull and anterior fontanelle were exposed. The mice were placed in a stereotactic frame. The coordinates of the anterior fontanelle were recorded. A cranial hole with a diameter of $1 \mathrm{~mm}$ was drilled at the position with coordinates (x-2.2 mm, y-0.2 mm). A needle was inserted into $3.5 \mathrm{~mm}$ of the right striatum. Collagenase ( $0.075 \mathrm{U}$ in $0.5 \mu \mathrm{L}$ phosphate buffered solution) was infused with a 10 $\mu \mathrm{L}$ microsyringe at a speed of $0.167 \mu \mathrm{L} / \mathrm{min}$. The needle was kept for $5 \mathrm{~min}$ after injection and then the needle was pulled out within $5 \mathrm{~min}$ at a constant speed. The hole was sealed with bone wax. Sham group were performed with the same operation except $0.5 \mu \mathrm{L}$ phosphate buffered solution was infused into the right striatum.

\subsection{Evaluation of neurological function}

The modified Garcia test was used to evaluate the neurological function of mice [23]. It contains seven different aspects: spontaneous activity in cage, response to vibrissae touch, response to sides of the abdomen's touch, symmetry of limbs, symmetry of movements, lateral turning, climbing ability. The highest score for each test is 3 , while the lowest score is 0 . The total score of modified Garcia test is 21 .

\subsection{Histological analysis of intestinal tissues}

At $24 \mathrm{~h}$ of $\mathrm{ICH}$, the animals were anesthetized by ketamine $(80 \mathrm{mg} / \mathrm{kg})$ and xylazine $(5$ $\mathrm{mg} / \mathrm{kg}$ ). The intestines were harvested and then they were fixed in $4 \%$ paraformaldehyde for 12 h. The tissues were embedded in paraffin wax and were cut into $5 \mu \mathrm{m}$ thick sections followed by hematoxylin and eosin staining. The images were taken with a microscope by an observer who is blinded to the experimental group. 


\subsection{Determination of the levels of ET}

Blood samples were obtained from the anesthetized mice. The blood was placed at room temperature for $30 \mathrm{~min}$ and centrifuged at $3500 \mathrm{rpm}$ for $15 \mathrm{~min}$ at $4^{\circ} \mathrm{C}$. According to the instruction of the company, the levels of ET was assayed with ELISA kits.

\subsection{Statistical analysis}

All data were expressed as mean \pm S.D. SPSS 23.0 software was used for data analysis. Significance differences were assessed with one-way ANOVA with the Bonferroni post hoc test for multiple $t$-tests. P value of $<0.05$ was considered statistically significant.

\section{Results}

\subsection{ATR, PHC and MLA affect the effect of ANP on neurological function in ICH mice}

Neurological function was evaluated at $24 \mathrm{~h}$ after ICH. As shown in Figure 1, compared with sham group, the neurological function of ICH was significantly decreased $(\mathrm{P}<0.01)$. ANP significantly improved the neurological function of ICH mice $(\mathrm{P}<0.01)$. No effect was observed when ATR was given to ICH mice $(\mathrm{P}>0.05)$. When ANP and ATR were administered simultaneously, the protective effect of ANP on the neurological function function was abated.

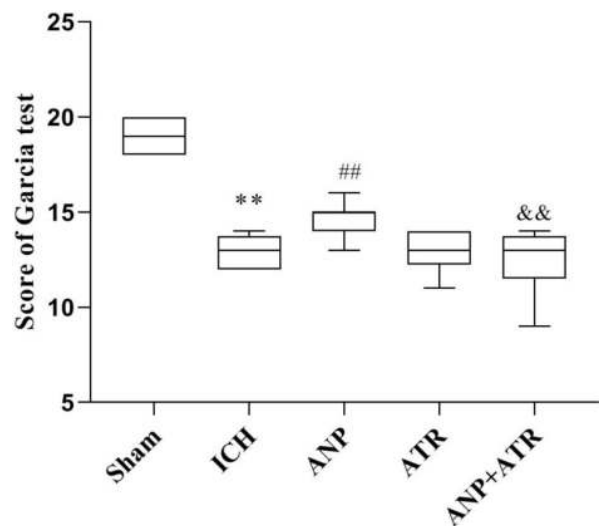

Figure 1 ATR affects the effect of ANP on neurological function in ICH mice. Data are expressed as means $\pm \mathrm{SD} ; \mathrm{n}=8$ in each group. ${ }^{* *} \mathrm{P}<0.01$ compared with sham group. ${ }^{\# \#} \mathrm{P}<0.01$ compared with ICH group; \&\& $\mathrm{P}<0.01$ compared with ANP group

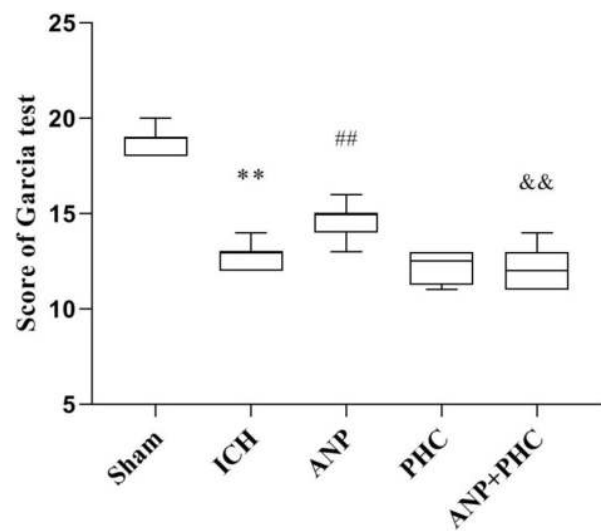

Figure 2 PHC affects the effect of ANP on neurological function in ICH mice. Data are expressed as meansSD; $\mathrm{n}=8$ in each group; ${ }^{* *} \mathrm{P}<0.01$ compared with sham group; $\# \# \mathrm{P}<0.01$ compared with ICH group; ${ }^{\&} \& \mathrm{P}<0.01$ compared with ANP group

As shown in Figure 2, compared with sham group, the neurological function of ICH was significantly decreased $(\mathrm{P}<0.01)$. ANP can significantly enhanced the neurological function of ICH mice $(\mathrm{P}<0.01)$. No effect was observed when $\mathrm{PHC}$ was given to ICH mice alone $(\mathrm{P}>$ 


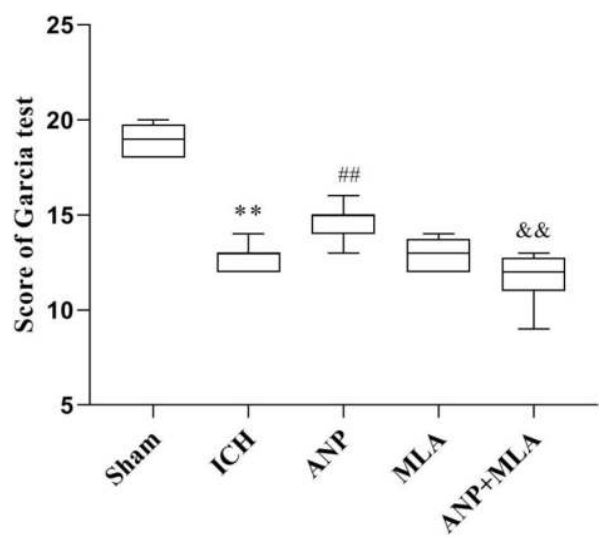

Figure 3 MLA affect the effect of ANP on neurological function in ICH mice. Data are expressed as means $\mathrm{SD} ; \mathrm{n}=8$ in each group; ${ }^{* *} \mathrm{P}<0.01$ compared with sham group; \#\# $\mathrm{P}<0.01$ compared with ICH group; \&\& $\mathrm{P}<0.01$ compared with ANP group

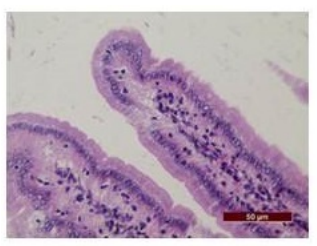

(a)

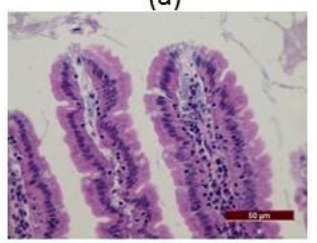

(d)

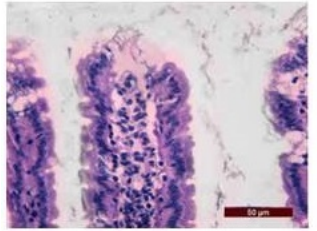

(g)

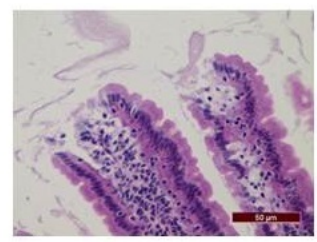

(b)

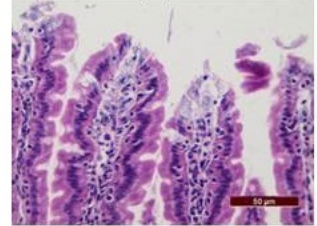

(e)

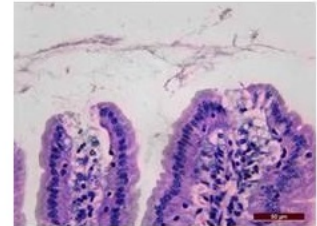

(h)

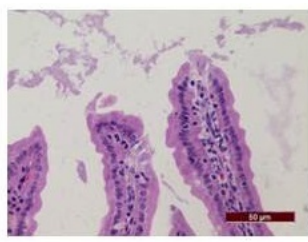

(c)

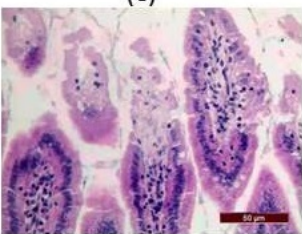

(f)

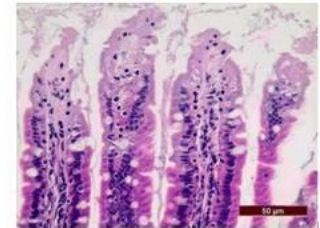

(i)

Figure 4 ATR, PHC and MLA affect the effect of ANP on intestinal injury in ICH mice. Tissues of intestine were stained with HE. (a) Sham group; (b) ICH group; (c) ANP group; (d) ANP+ATR group; (e) ANP + PHC group; (f) ANP + MLA group; (g) ATR group; (h) PHC group; (i) MLA group. ( $\mathrm{n}=3$ in each group, magnification, 400 )

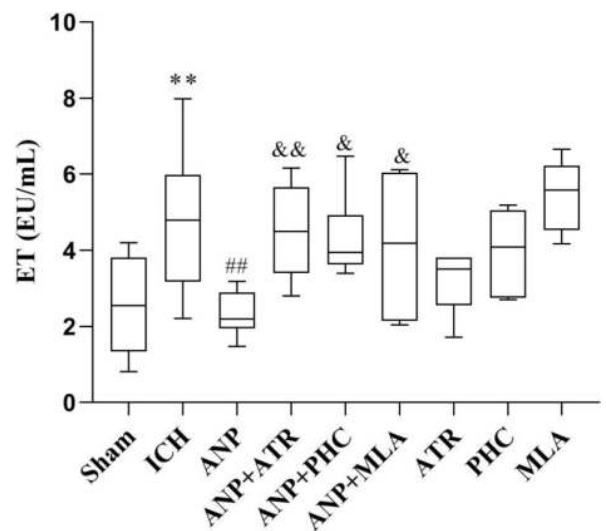

Figure 5 Effects of ANP, ATR, PHC and MLA on ET level in serum. Data are expressed as means \pm SD $\mathrm{n}=6$ in each group; ${ }^{* *} \mathrm{P}<0.01$ compared with Sham group; \#\# $\mathrm{P}<0.01$ compared with ICH group; \& $\mathrm{P}$ $<0.05,{ }^{\& \&} \mathrm{P}<0.01$ compared with ANP group 
0.05). When ANP and PHC were given to ICH mice simultaneously, the protective effect of ANP on the neurological function of ICH mice was abrogated.

As shown in Figure 3, compared with sham group, the neurological function of ICH was significantly decreased $(\mathrm{P}<0.01)$. ANP significantly improved the neurological function of ICH mice $(\mathrm{P}<0.01)$. No effect was observed when MLA was given to ICH mice alone ( $\mathrm{P}$ $>0.05$ ). When ANP and MLA were given to ICH mice simultaneously, the protective effect of ANP on the neurological function of ICH mice was abated.

\subsection{ATR, PHC and MLA affect the effect of ANP on intestinal injury in ICH mice}

According Figure 4, histopathological examination showed that morphology of the intestinal mucosa was intact in the sham group. The epithelial cells at the top of the intestinal villi in the ICH group were exfoliated and necrotic. ANP reduced the ICH-induced intestinal injury. Compared with ICH group, ATR, PHC and MLA have no influence on intestine injury. However, ATR, PHC or MLA partially abrogated the ANP-mediated protective effect in the intestine of ICH mice.

\subsection{Effects of ANP, ATR, PHC and MLA on ET level in serum}

According to Figure 5, the ET concentration in serum was higher in the ICH group than that in sham group $(\mathrm{P}<0.01)$. This increase was significantly reduced by ANP administration ( $\mathrm{P}$ $<0.01)$. When given ATR, PHC or MLA, the concentration of ET did not show significant changes compared with ICH group $(\mathrm{P}>0.05)$. When given ATR, PHC or MLA with ANP, the ANP-mediated decrease of ET was partially abrogated $(\mathrm{P}<0.05)$.

\section{Discussion and conclusions}

Stroke is a stress, which leads to dysfunction of the nervous system. Stroke-induced inhibition in parasympathetic nervous system is always companied by intestine injury [24]. Stanley and colleagues reported that microbes in host small intestine play a key role in the poststroke infection [25]. The gastrointestinal function is important for multiple organ dysfunction syndrome in stroke patients. The relationship between intestinal protection and neurological function remains to be further studied.

ANP, a Traditional Chinese Medicine, has been widely used in China to treat fever, coma, stupor, hemorrhage, and so on. Clinical researches use ANP as an adjuvant therapy in stroke patients, which has proven to improve neurological function and to shorten coma time [20]. Previous study shows that ANP has a protective effect on neurological function and intestine tissue. This study investigates whether parasympathetic nerve is involved in the process of ANP action. ICH can cause neurological dysfunction and intestinal injury in mice [26]. Previous study of our laboratory showed that ANP improved neurological dysfunction in ICH. Intestinal injury also can be alleviated by ANP treatment [21]. Stroke causes a stress response in the body, and the parasympathetic nervous system is suppressed. It is still not clear whether ANP can regulate the parasympathetic nervous system and thus exerts a protective effect on ICH.

The intestinal function of ICH has attracted much attention because the intestine is the central organ of stress in the body. It also plays an important role in the formation of multi-organ dysfunction syndrome [27]. The results showed that the intestinal mucosa of the ICH mice had changes of villous structure, and some villous epithelial cells were denatured and necrosed. Under the stress of ICH, blood flow is redistributed. Gastrointestinal blood flow is reduced and ischemia occurs, which directly causes intestinal mucosal cell atrophy, shedding and necrosis.

It is well known that parasympathetic system is governed by acetylcholine. Acetylcholine receptors contain muscarinic $(\mathrm{M})$ receptors and nicotinic $(\mathrm{N})$ receptors. $\mathrm{M}$ receptors have five subtypes, $\mathbf{M}_{1}-\mathrm{M}_{5}$. They are widely distributed in central and peripheral nervous system $[28,29]$. $M_{2}$ receptor are widely distributed in heart [29]. The expression of $M_{1}$ and $M_{3}$ receptors is higher than $\mathrm{M}_{2}$ in intestine [30].

ATR is a non-selective $\mathrm{M}$ receptor antagonist. ATR blocks peripheral and central $\mathrm{M}$ cholinergic receptors [31]. A large dose of ATR excites central nervous system, and the increase of the ATR dose will produce central poisoning symptoms or even death. ATR inhibited the motor function of the small intestine [31]. The present results indicated that intestinal tissue of ICH mice was damaged. Using ATR alone does not affect intestinal tissue damage in mice. ANP attenuates intestinal tissue damage in ICH mice. ATR weaken the protective effect of ANP on 
the intestinal tissue of ICH mice. It suggested that ATR may reduce the protective effect of ANP on the intestinal tissue of ICH mice by blocking $\mathrm{M}$ receptor.

PHC is a selective $\mathrm{M}_{1}, \mathrm{M}_{3}$ and $\mathrm{N}$ receptor antagonist [33]. It has anti-cholinergic effect on the central and peripheral nerves, and less side effects to cardiovascular system. PHC did not affect the motor function of the small intestine [32]. PHC not only blocks $\mathrm{N}_{1}$ receptor in nerves centralis, but also blocks $\mathrm{M}_{3}$ receptor in small intestinal to relax small intestinal smooth muscles. PHC blocks parasympathetic excitation caused by the release of acetylcholine. The $M_{3}$ receptor is widely distributed in the small intestine. When the $\mathrm{M}_{3}$ receptor is inhibited, the movement of the small intestine slows down, causing small intestinal damage. As can be seen from these experimental results, the intestinal tissue of ICH mice was injured. Using PHC alone did not affect intestinal tissue damage in mice. ANP improved intestinal tissue damage in ICH mice. The effect of ANP on intestinal tissue protection in ICH mice was partially abated. These results suggest that PHC can reduce the protective effect of ANP on the intestine in ICH mice by blocking the $\mathrm{M}_{3}$ receptor.

MLA is an acetylcholine 7 nicotinic receptor $(\alpha 7 \mathrm{nAChR})$ antagonist. $\alpha 7 \mathrm{nAChR}$ plays a key role in inhibiting cytokine synthesis through the cholinergic anti-inflammatory pathway (CAP) [14]. Activation of the endogenous CAP leads to an increased release of acetylcholine (Ach) from the vagus nerve. Ach binds to specific receptors on the surface of macrophages and exerts its anti-inflammatory effect by inhibiting the release of inflammatory mediators. Previous study demonstrates that Ach reduces the release of tumor necrosis factor, interleukin (IL-1, IL-6, and IL-18) in LPS-stimulated human macrophage culture [13]. In this study, using MLA alone did not affect intestinal tissue damage in ICH mice. ANP ameliorated the intestinal tissue damage in ICH mice. MLA abrogated the protective effect of ANP on the intestinal tissue in ICH mice. These results suggest that MLA may reduce the protective effect of ANP on intestine tissues in ICH mice by blocking peripheral CAP receptor. $\mathrm{N}$ receptor (especially $\alpha 7 \mathrm{nAChR}$ ) might participate in the protective effect of ANP on neurological function and intestinal injury in ICH mice.

In brief, it is reasonable to conclude that ANP has protective effect on neurological function and intestinal tissues in ICH mice, and its mechanism may be related to the regulation of $\mathrm{M}_{3}$ receptor and the $\mathrm{N}$ receptor. Parasympathetic nerve system is involved in the protective effect of ANP on neurological function and intestinal tissues in ICH mice.

The concentrations of ET in plasma is an important index of intestinal dysfunction. When intestine is damaged, ET enter the bloodstream, causing further damage to intestinal function. In other word, increasing concentrations of ET indicate severe intestinal barrier dysfunction [34]. The concentration of ET in the blood can reflect the permeability of intestinal mucosa and the function of intestinal mucosal barrier. Intestinal microbes-derived ET is an important internal factor in central nervous inflammation. It is a potent activator of the immune and inflammatory systems, which leads to the increase of TNF- $\alpha$ concentration [35]. Macrophages and epithelial cells, produce TNF- $\alpha$ during bacterial infection or ET stimulation. It initiates inflammatory response by promoting the release of other inflammatory factors and cytokines [36]. Research shows that ET can induced neuroinflammation and neurological disorder [37]. The concentration of ET in the blood of the ICH mice is significantly increased. ANP can reduce the concentration of ET in the blood. ATR, MLA and PHC administration alone did not affect the ET content. However, ATR, MLA and PHC can abate the ANP-mediated changes of ET in the blood of ICH mice. Based on the findings in our study, we speculate that the protection of ANP on neurological function in ICH mice may be associated with the change of ET concentration in circulation.

According to these results, $\mathrm{M}$ and $\mathrm{N}$ receptor blockers abrogate the protective effect of ANP on neurological function and intestinal injury in $\mathrm{ICH}$ mice. $\mathrm{M}_{3}$ and $\mathrm{N}$ receptor (especially $\alpha 7$ nAChR blocker) may participate in the protective effect of ANP in ICH mice. Therefore, we conclude that ANP improves the neurological function and protects the intestinal tissue in ICH mice by regulating the parasympathetic nerve system. However, it should be noted that whether ANP exerts its action by regulating the levels of transmitters or the expression of cholinergic receptors is still unclear. Future study should focus on investigating the content mentioned above.

\section{Authors contributions}

Fenghua Fu conceived the present study and reviewed the manuscript. Yunqi Yang, Shumin Yue, Mingan $\mathrm{Li}$, and Lin Zhou performed the experiments. Yunqi Yang and Tian Wang analyzed the data and drafted the manuscript. All authors approved the submission. 


\section{Conflict of interest}

The authors declare no potential conflicts of interest with respect to this report, authorship, and/or publication of this article.

\section{Funding}

This work was supported by National Natural Science Foundation of China (No. 81873039).

\section{References}

[1] Van Asch CJJ, Luitse MJA, Rinkel GJE, et al. Incidence, case fatality, and functional outcome of intracerebral haemorrhage over time, according to age, sex, and ethnic origin: a systematic review and meta-analysis. The Lancet Neurology, 2010, 9(2): 167-176. https://doi.org/10.1016/s1474-4422(09)70340-0

[2] Zhou M, Wang H, Zeng X, et al. Mortality, morbidity, and risk factors in China and its provinces, 1990-2017: a systematic analysis for the Global Burden of Disease Study 2017. The Lancet, 2019, 394(10204): 1145-1158. https://doi.org/10.1016/s0140-6736(19)30427-1

[3] Wang J, Zhang X, Yang C, et al. Effect of monoacylglycerol lipase inhibition on intestinal permeability in chronic stress model. Biochemical and Biophysical Research Communications, 2020, 525(4): 962 967. https://doi.org/10.1016/j.bbrc.2020.02.173

[4] Ji R, Shen H, Pan Y, et al. A novel risk score to predict 1-year functional outcome after intracerebral hemorrhage and comparison with existing scores. Critical Care (London, England), 2013, 17(6): $\mathrm{R} 275$.

https://doi.org/10.1186/cc13130

[5] Hannawi Y, Hannawi B, Rao CP, et al. Stroke-associated pneumonia: major advances and obstacles Cerebrovascular Diseases (Basel, Switzerland), 2013, 35(5): 430-443. https://doi.org/10.1159/000350199

[6] Poon MT, Fonville AF and Al-Shahi Salman R. Long-term prognosis after intracerebral haemorrhage: systematic review and meta-analysis. Journal of Neurology, Neurosurgery, and Psychiatry, 2014 85(6): 660-667. https://doi.org/10.1136/jnnp-2013-306476

[7] Flaherty ML, Haverbusch M, Sekar P, et al. Long-term mortality after intracerebral hemorrhage. Neurology, 2006, 66(8): 1182-1186. https://doi.org/10.1212/01.wnl.0000208400.08722.7c

[8] Kalaria RN, Akinyemi R and Ihara M. Stroke injury, cognitive impairment and vascular dementia. Biochimica et Biophysica Acta (BBA) - Molecular Basis of Disease, 2016, 1862(5): 915-925. https://doi.org/10.1016/j.bbadis.2016.01.015

[9] Iglesias-Rey R, Rodríguez-Yáñez M, Arias S, et al. Inflammation, edema and poor outcome are associated with hyperthermia in hypertensive intracerebral hemorrhages. European Journal of Neurology, 2018, 25(9): 1161-1168. https://doi.org/10.1111/ene.13677

[10] Zhou Y, Wang Y, Wang J, et al. Inflammation in intracerebral hemorrhage: from mechanisms to clinical translation. Progress in Neurobiology, 2014, 115: 25-44. https://doi.org/10.1016/j.pneurobio.2013.11.003

[11] De Raedt S, De Vos A and De Keyser J. Autonomic dysfunction in acute ischemic stroke: an underexplored therapeutic area? Journal of the Neurological Sciences, 2015, 348(1-2): 24-34. https://doi.org/10.1016/j.jns.2014.12.007

[12] Shoup JP, Winkler J, Czap A, et al. beta-Blockers associated with no class-specific survival benefit in acute intracerebral hemorrhage. Journal of the Neurological Sciences, 2014, 336(1-2): 127-131. https://doi.org/10.1016/j.jns.2013.10.022

[13] Borovikova LV, Ivanova S, Zhang M, et al. Vagus nerve stimulation attenuates the systemic inflammatory response to endotoxin. Nature, 2000, 405(6785): 458-462.

[14] Wang H, Yu M, Ochani M, et al. Nicotinic acetylcholine receptor alpha7 subunit is an essential regulator of inflammation. Nature, 2003, 421(6921): 384-388.

[15] Swor DE, Thomas LF, Maas MB, et al. Admission Heart Rate Variability is Associated with Fever Development in Patients with Intracerebral Hemorrhage. Neurocritical Care, 2019, 30(2): 244-250. https://doi.org/10.1007/s12028-019-00684-w

[16] Fernández-Cabezudo MJ, George JA, Bashir G, et al. Involvement of Acetylcholine Receptors in Cholinergic Pathway-Mediated Protection Against Autoimmune Diabetes. Frontiers in Immunology, 2019, 10: 1038 . https://doi.org/10.3389/fimmu.2019.01038 
[17] Zhang J, Zhang L, Sun X, et al. Acetylcholinesterase Inhibitors for Alzheimer's Disease Treatment Ameliorate Acetaminophen-Induced Liver Injury in Mice via Central Cholinergic System Regulation. The Journal of Pharmacology and Experimental Therapeutics, 2016, 359(2): 374-382. https://doi.org/10.1124/jpet.116.233841

[18] Zhao L, Peng F, Guan B, et al. Whether Metal Element-Containing Herbal Formula Angong Niuhuang Pill Is Safe for Acute Brain Disorders? Biological Trace Element Research, 2015, 166(1): 41-48. https://doi.org/10.1007/s12011-015-0318-3

[19] Guo Y, Yan S, Xu L, et al. Use of angong niuhuang in treating central nervous system diseases and related research. Evidence-based Complementary and Alternative Medicine, 2014: 346918. https://doi.org/10.1155/2014/346918

[20] Liu H, Yan Y, Pang P, et al. Angong Niuhuang Pill as adjuvant therapy for treating acute cerebral infarction and intracerebral hemorrhage: A meta-analysis of randomized controlled trials. Journal of Ethnopharmacology, 2019, 237: 307-313. https://doi.org/10.1016/j.jep.2019.03.043

[21] Wu S, Lv H, Wang W, et al. Effects of Angong Niuhuang Pill on intestinal mucosal barrierin intracerebral hemorrhage mice. Pharmacology and Clinics of Chinese Materia Medica. 2018, 34(01):610.

[22] Klebe D, Iniaghe L, Burchell S, et al. Intracerebral Hemorrhage in Mice. Methods in Molecular Biology (Clifton, N.J.), 2018, 1717: 83-91. https://doi.org/10.1007/978-1-4939-7526-6_7

[23] Garcia JH, Wagner S, Liu KF, et al. Neurological deficit and extent of neuronal necrosis attributable to middle cerebral artery occlusion in rats. Statistical validation. Stroke, 1995, 26(4): 627-635. https://doi.org/10.1161/01.str.26.4.627

[24] Novosad VL, Richards JL, Phillips NA, et al. Regional susceptibility to stress-induced intestinal injury in the mouse. American Journal of Physiology-Gastrointestinal and Liver Physiology, 2013, 305(6): G418-G426. https://doi.org/10.1152/ajpgi.00166.2013

[25] Stanley D, Mason LJ, Mackin KE, et al. Translocation and dissemination of commensal bacteria in post-stroke infection. Nature Medicine, 2016, 22(11): 1277-1284. https://doi.org/10.1038/nm.4194

[26] Cheng Y, Zan J, Song Y, et al. Evaluation of intestinal injury, inflammatory response and oxidative stress following intracerebral hemorrhage in mice. International Journal of Molecular Medicine, 2018, 42(4): 2120-2128. https://doi.org/10.3892/ijmm.2018.3755

[27] Klingensmith NJ, Coopersmith CM. The Gut as the Motor of Multiple Organ Dysfunction in Critical Illness. Critical Care Clinics, 2016, 32(2): 203-212. https://doi.org/10.1016/j.ccc.2015.11.004

[28] Lebois EP, Thorn C, Edgerton JR, et al. Muscarinic receptor subtype distribution in the central nervous system and relevance to aging and Alzheimer's disease. Neuropharmacology, 2018, 136(Part C): $362-373$. https://doi.org/10.1016/j.neuropharm.2017.11.018

[29] Saternos HC, Almarghalani DA, Gibson HM, et al. Distribution and function of the muscarinic receptor subtypes in the cardiovascular system. Physiological Genomics, 2018, 50(1): 1-9. https://doi.org/10.1152/physiolgenomics.00062.2017

[30] Tangsucharit P, Takatori S, Zamami Y, et al. Muscarinic acetylcholine receptor $\mathrm{M}_{1}$ and $\mathrm{M}_{3}$ subtypes mediate acetylcholine-induced endothelium-independent vasodilatation in rat mesenteric arteries. Journal of Pharmacological Sciences, 2016, 130(1): 24-32. https://doi.org/10.1016/j.jphs.2015.12.005

[31] Wang Y, Lin D, Tan H, et al. Penehyclidine hydrochloride preconditioning provides pulmonary and systemic protection in a rat model of lung ischaemia reperfusion injury. European Journal of Pharmacology, 2018, 839: 1-11. https://doi.org/10.1016/j.ejphar.2018.09.012

[32] Liu Z, Wen G, Hua W, et al. Comparision of the effect of penehyclidine hydrochloride and atropine on gastrointestinal hormone in mice. Journal of Clinical Anesthesiology, 2012, 28(07): 25-44.

[33] Shu Y, Yang Y and Zhang P. Neuroprotective effects of penehyclidine hydrochloride against cerebral ischemia/reperfusion injury in mice. Brain Research Bulletin, 2016, 121: 115-123. https://doi.org/10.1016/j.brainresbull.2016.01.008

[34] Singleton KD and Wischmeyer PE. Oral glutamine enhances heat shock protein expression and improves survival following hyperthermia. Shock. 2006, 25(3): 295-299. https://doi.org/10.1097/01.shk.0000196548.10634.02

[35] Sun J, Li F, Chen J, et al. Effect of ketamine on NF-kappa B activity and TNF-alpha production in endotoxin-treated rats. Annals of Clinical and Laboratory Science, 2004, 34(2): 181-186.

[36] Bahramabadi R, Fathollahi MS, Hashemi SM, et al. Serum Levels of IL-6, IL-8, TNF- $\alpha$, and TGF- $\beta$ in Chronic HBV-Infected Patients: Effect of Depression and Anxiety. Laboratory Medicine, 2017, 49(1): 41-46. https://doi.org/10.1093/labmed/lmx064

[37] Lopes PC. LPS and neuroinflammation: a matter of timing. Inflammopharmacology, 2016, 24(5): 291-293. https://doi.org/10.1007/s10787-016-0283-2 